\title{
Hemifacial microsomia: management of the vertical ramus compartment
}

\author{
Maurice Yves Mommaerts \\ European Face Centre, Universitair Ziekenhuis Brussel, Laarbeeklaan 101, 1090 Brussels, Belgium.
}

Address for correspondence: Prof. Maurice Yves Mommaerts, European Face Centre, Universitair Ziekenhuis Brussel, Laarbeeklaan 101, 1090 Brussels, Belgium. E-mail: maurice.mommaerts@uzbrussel.be

\begin{abstract}
Hemifacial microsomia and Goldenhar syndrome pose unique challenges to the craniofacial surgeon. The O.M.E.N.S. classification provides a description of the craniofacial features. For the " $\mathrm{M}^{\text {" of }}$ O.M.E.N.S. (the mandible), the Pruzansky-Kaban classification provides therapeutic guidelines for joint and face reconstruction. A sequence of standard procedures, including temporomandibular joint reconstruction, facial rotation surgery, gluteal fat grafting, and patient-specific titanium implantation, each have their intricacies. The author provides his expert opinion, acquired over thirty years of experience, with an emphasis on descriptions of and solutions for ten problematic issues.
\end{abstract}

Key words:

Congenital abnormalities, goldenhar syndrome, mandibular reconstruction

\section{INTRODUCTION}

Hemifacial microsomia is the second most common facial birth disorder, with a prevalence of one in 3,500-6,000 live births. ${ }^{[1]}$ In $70 \%$ of individuals, the condition is unilateral [Figure 1]. The "O.M.E.N.S." acronym is the most commonly used way to categorize hemifacial microsomia. This acronym stands for orbital, mandibular, ear, facial nerve, and soft tissue deficiencies, which are rated on a scale of $0-3$, according to their severity. ${ }^{[2]}$ Most striking upon clinical examination are the external ear deformities [Figure 2] and the facial asymmetry. The latter is related to deficiencies in the vertical ramus compartment, originating from both skeletal tissues (mandible and skull base) and soft tissues (muscles of mastication and subcutaneous fat) [Figure 3]. The mandibular deformity, considered separately from the skull base (temporal bone and orbit) deformities, has been classified by Pruzansky and Kabanas Type I to III $^{[3,4]}$ [Figures 4-7].

\begin{tabular}{|l|l|}
\hline \multicolumn{2}{|c|}{ Access this article online } \\
\hline Quick Response Code: & Website: \\
\hline & www.parjournal.net \\
\cline { 2 - 3 } & \\
\hline & DOI: \\
\hline & $10.4103 / 2347-9264.157097$ \\
\hline
\end{tabular}

From the mid-1970s to the mid-1990s, treatment modalities for Type I and Iia Pruzansky-Kaban mandibular deformities included orthognathic treatment during adolescence or "functional" orthodontic appliances ${ }^{[5]}$ and early mandibular osteotomies to keep pace with the rate of vertical midfacial growth. ${ }^{[6]}$ For Type IIb and III deformities in growing children, joint reconstruction with costochondral grafting was indicated. In the mid-1990s, early distraction osteogenesis, before skeletal maturation and/or permanent dentition, was believed to induce the formation of not only bone, but also of soft tissue. However, a study published in $2002^{[7]}$ and a systematic review published in $2009^{[8]}$ concluded that there are no long-term benefits to early osteodistraction in the vertical ramus.

The aim of this article was to explain the author's protocol for the reconstruction of the vertical ramus compartment in hemifacial microsomia, highlighting the key issues of the technique. All patients involved in this article agreed to publish their facial pictures and signed the consent form.

\section{KEY ASPECTS OF SURGERY}

To illustrate the author's treatment strategy for a deficiency of the vertical ramus compartment, 10 salient points are presented with illustrative photographs from a series of patients. The general approach for the different Pruzansky-Kaban types is presented in Table 1. Orbito-zygomatic and jaw angle reconstructions are performed in all types of hemifacial microsomia 


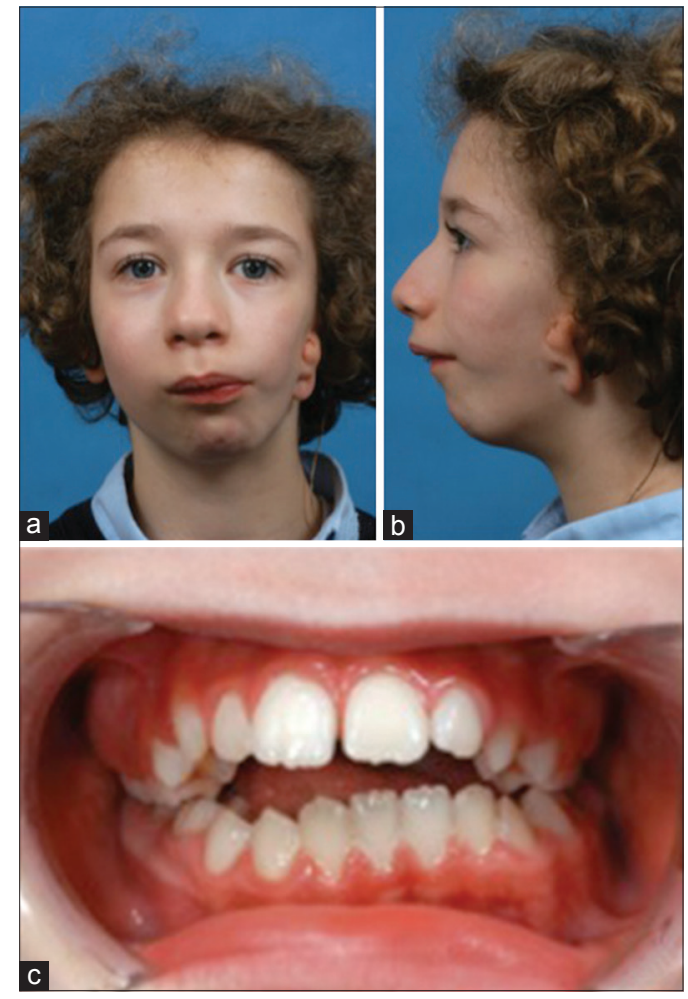

Figure 1: An O0 M2a E2 NO S2 case. (a) Frontal view; (b) left profile view; (c) frontal occlusion view

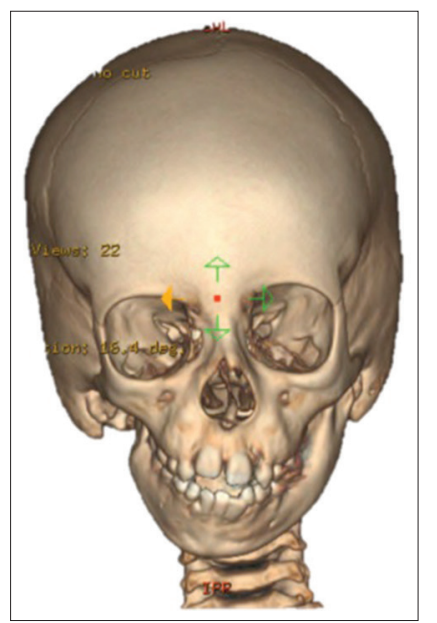

Figure 3: Three-dimensional (3D) reconstruction of a multi-slice computed tomography (CT) scan of the skull of an O1M2b, E1, N0, S1 case of hemifacial microsomia. The skeletal asymmetry in this case is due to the absence of the right-sided ascending ramus, with compensating downward growth of the skull base and orbit at the affected side

following the facial rotation procedure. Hard and soft tissue volume deficiencies can be addressed by free gluteal fat transplantation, three-dimensional (3D) printed patient-specific titanium implants or a combination of both.

\section{Point I: Skeletal symmetrization increases left/} right soft tissue volume discrepancy

The facial rotation procedure,$^{[9]}$ consisting of rotation of the maxillary, mandibular, and chin segments around a sagittal axis, while translating the midlines of all segments to the predetermined facial midline and advancing the lower face to the ideal facial profile in a sagittal plane,

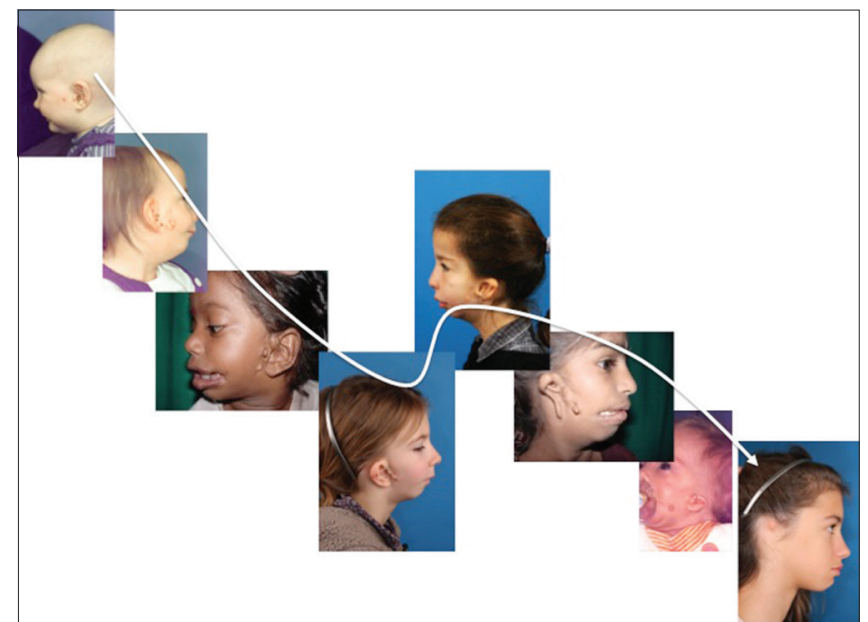

Figure 2: Ear deformities from 0 to 3 dysmorphic severity, as indicated by the white arrow

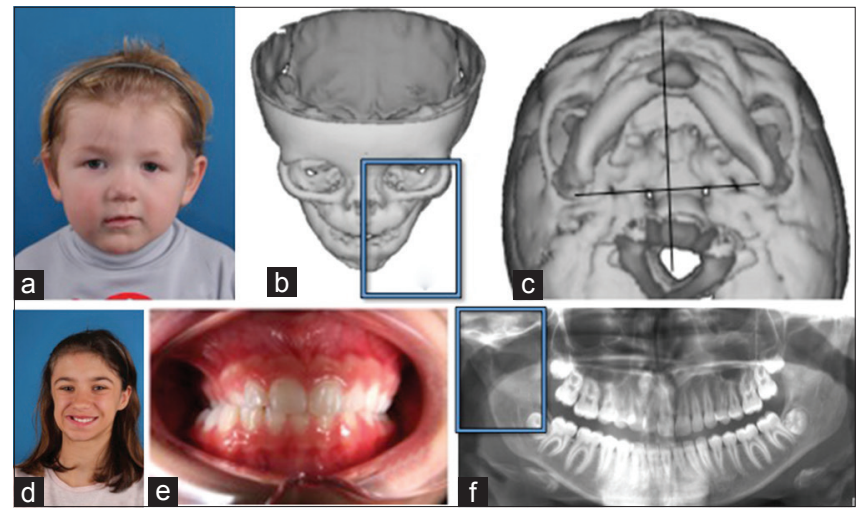

Figure 4: Pruzansky-Kaban Type I. All mandibular and temporomandibular joint components are present and normal in shape, but they are hypoplastic to a variable degree, compared to the contralateral side. (a) Frontal facial view of an affected girl during childhood; (b) three-dimensional (3D) reconstruction of a multi-slice computed tomography (CT) scan of the skull of a Type I deformity, with deviation of the mandibular midline to the left; (c) submentovertical projection of the same 3D CT scan, demonstrating mandibular asymmetry; (d) frontal view of an affected girl during adolescence; (e) frontal view of the occlusion of the same girl in (d), demonstrating cross-bite on the right; (f) orthopantomogram of the girl in (d), showing the joint structures with a normal shape and location, but with a degree of hypoplasia. Note the downward growth of the skull base on the affected side

results in displacement of hard and soft tissues to the normal side [Figures 8-10]. This results in additional asymmetry when the left and right sides are mirrored, necessitating the next procedure: compensating for the volume deficit.

\section{Point 2: Early osteodistraction of the horizontal ramus}

Prior to orthodontic decompensation, at the age of $11-12$, it is necessary to judge the retromolar bone stock. In view of the upcoming sagittal split osteotomy with the substantial advancement of the affected side, a decision must be made between removal of impacted second and/or third molars or osteodistraction of the horizontal ramus. Osteodistraction not only creates more bone to work with, but it can also partially correct the horizontal deficiency [Figure 11]. The fibrous tissues of the vertical ramus compartment can 


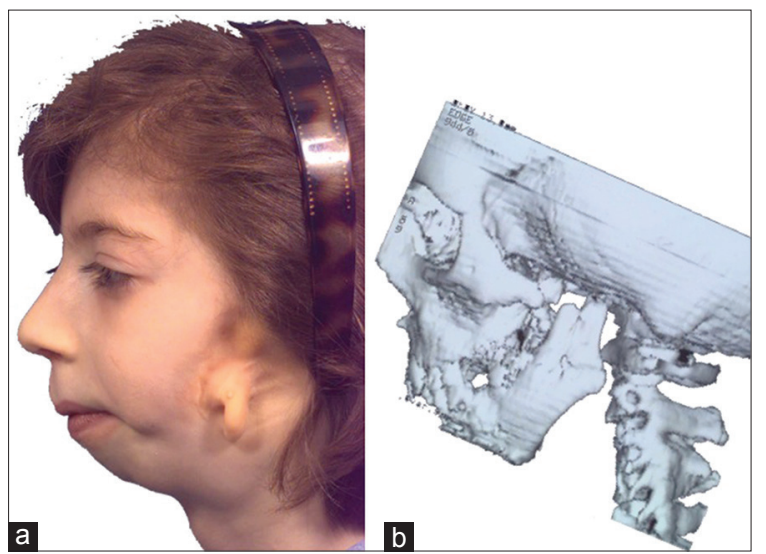

Figure 5: Pruzansky-Kaban Type IIa. The mandibular ramus, condyle, and temporomandibular joint are present but hypoplastic and abnormal in shape. The mouth can be symmetrically opened. (a) Profile view of the affected side in an adolescent girl; (b) three-dimensional (3D) reconstruction of a multi-slice computed tomography (CT) scan of the viscero-cranium of the patient in a, demonstrating the abnormal shape and hypoplasia of the vertical ramus of the mandible

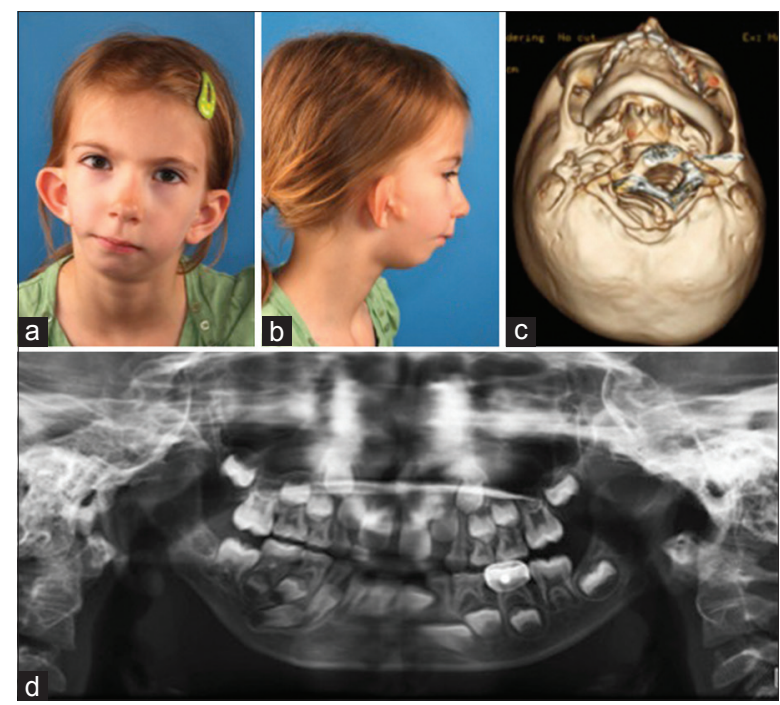

Figure 6: Pruzansky-Kaban Type IIb. The mandibular ramus is hypoplastic and markedly abnormal in form and location, being medial, anterior and inferior. There is no articulation with the temporal bone. (a) Frontal view of an affected adolescent; (b) profile view of the affected side of the girl in (a); (c) three-dimensional (3D) reconstruction of a multi-slice computed tomography (CT), submento-vertical view, demonstrating the abnormal structures in abnormal location (the same patient as in (a) and (b)); (d) orthopantomogram of the girl in (a), (b), and (c), showing downward growth of the skull base on the affected side and no articulation

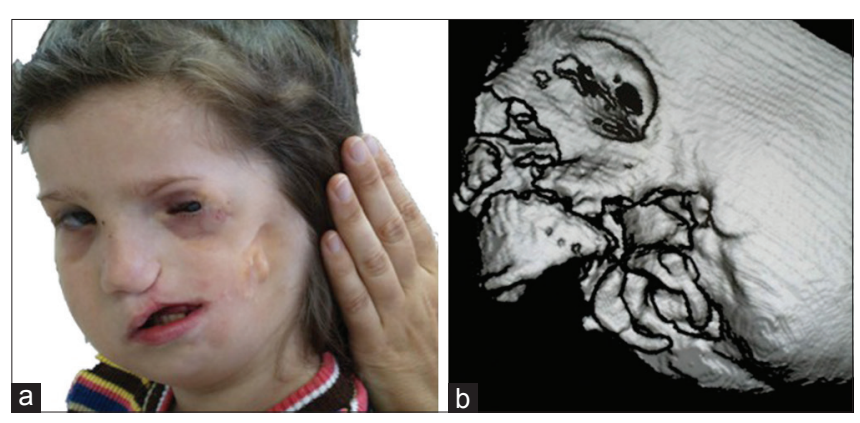

Figure 7: Pruzansky-Kaban Type III. The mandibular ramus, condyle, and temporomandibular joint are absent. The lateral pterygoid muscle and temporalis muscle, if present, are not attached to the mandibular remnant. (a) Three-fourths profile view of an affected girl; (b) three-dimensional (3D) reconstruction of a multi-slice computed tomography (CT) scan of the same girl as in a, showing the absence of the vertical ramus. The patient also had a unilateral cleft lip, alveolus, and palate
Table 1: General treatment strategies based on the Pruzansky-Kaban classification of mandibular abnormalities

\begin{tabular}{ll}
\hline $\begin{array}{l}\text { Pruzansky-Kaban } \\
\text { type }\end{array}$ & Treatment strategies \\
\hline Type I & $\begin{array}{l}\text { Orthognathic surgical correction "facial } \\
\text { rotation"[9] after orthodontic alignment, } \\
\text { coordination and decompensation. Standard } \\
\text { le Fort I, bilateral sagittal split osteotomies, } \\
\text { and sliding genioplasty techniques are used } \\
\text { Surgery is only performed early at the } \\
\text { age of } 4 \text { and older, when there is a centric } \\
\text { occlusion-centric relation shift of more } \\
\text { than } 5 \text { mm. The surgery involves joint } \\
\text { reconstruction with costochondral grafting } \\
\text { Osteodistraction in the horizontal (not } \\
\text { vertical) ramus is performed when there is } \\
\text { insufficient bone stock to perform a sagittal } \\
\text { split osteotomy after puberty } \\
\text { Orthognathic surgical correction "facial } \\
\text { rotation" after orthodontic alignment, } \\
\text { coordination, and decompensation is } \\
\text { performed at puberty and later } \\
\text { Joint and ramus reconstruction at the age of } \\
4 \text { and older. Orthognathic surgical correction } \\
\text { "facial rotation" after orthodontic alignment, } \\
\text { coordination and decompensation, at } \\
\text { puberty and later }\end{array}$ \\
\hline
\end{tabular}

be stretched more easily when the process occurs gradually. After the latter procedure, vertical ramus lengthening is easier to perform, as more bony overlap allows for more stable osteosynthesis and improved healing.

\section{Point 3: Choice of the pivot}

Rotation of the maxilla around a sagittal axis determines the correction of the occlusal plane cant and helps to swing the mandible to the midline [Figure 12]. The dental midlines are translated toward the healthy side for alignment with the predetermined facial midline. Finally, the chin point is adjusted in a translational way to correct the skeletal mandibular midline. The chin point is often also rotated along a sagittal axis to deal with the symphyseal height difference.

Disimpacting the affected side necessitates a bone graft, obtained from the calvarium or iliac crest. Impacting the healthy side does not stretch the fibrous remains of the masticatory muscles on the other side and is possible only when a gummy smile exists on that side [Figure 13]. The decision for the pivot relates to aesthetic desiderata (normal tooth-to-incisor distance and limited gummy smile) and functional desiderata (anti-relapse biomechanics). Hence, the pivot can be located at one of three positions: at the zygomatic buttress of the healthy side, at the zygomatic buttress of the affected side, or at the nasal spine. Pivoting at the affected side (and hence impacting at the healthy side) provides the least risk of relapse.

\section{Point 4: Skeletal suspension}

Skeletal suspension is mandatory to control the correction of the occlusal plane cant and the dental midline during healing [Figure 14]. As most cases of 


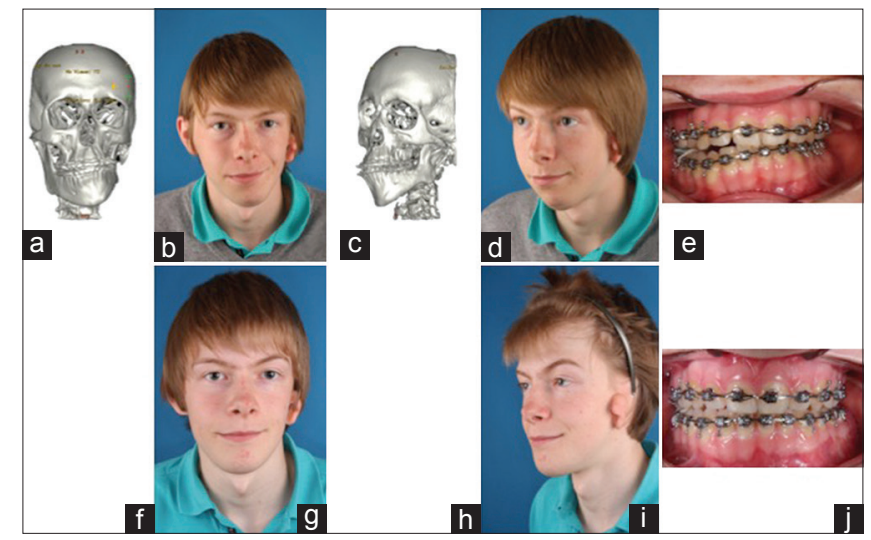

Figure 8: Pruzansky-Kaban Type IIa. (a-e) Situation prior to the facial rotation procedure; $(\mathrm{f}-\mathrm{j})$ situation after the primary skeletal and occlusal correction, demonstrating an increased left to right volume difference

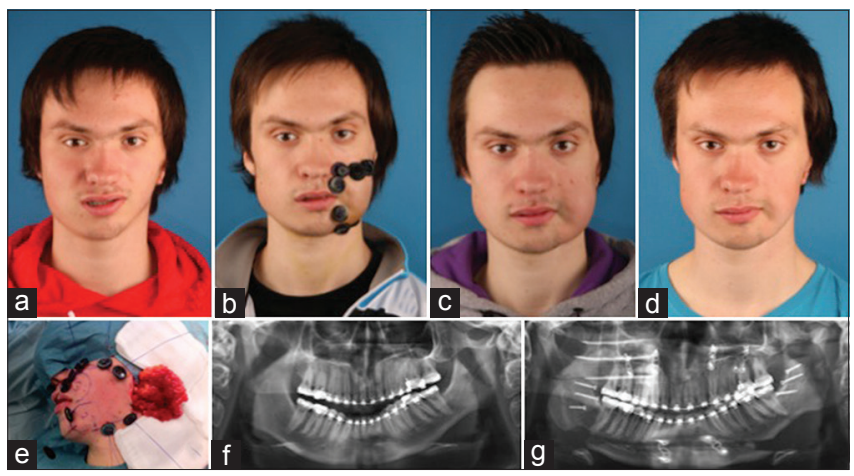

Figure 10: Pruzansky-Kaban Type Ila. (a) Frontal view after orthodontic preparation; (b) frontal view after facial rotation and free fat grafting; (c) frontal view six months after free fat grafting; (d) frontal view one year after free fat grafting; (e) gluteal fat tailored after facial requirements, ready to be inserted via a "short scar facelift" incision; (f) orthopantomogram after orthodontic preparation; (g) orthopantomogram immediately after the facial rotation procedure (note the massive chin osteotomy displacement)

hemifacial microsomia are unilateral, the rotational movement leads to different relapse vectors at both sides. Interarch elastics will safeguard the occlusal relationships, but not the skeletal relationships. The focus of interest is the occlusal plane and the lower dental midline. An orthodontic bone anchor or piriform aperture suspension wire(s) provide a means to suspend the rotated mandible with postoperative elastics to a stable osseous midface structure. Suspending the mandible to the repositioned maxilla is not sufficient, as it may give way and derotate.

\section{Point 5: The reference plane}

The oculo-auriculo-vertebral spectrum encompasses both hemifacial microsomia and Goldenhar syndrome. In addition to the aforementioned features of hemifacial microsomia, individuals with Goldenhar syndrome may exhibit ocular dermoid cysts, coloboma in the upper eyelids, delayed tooth eruption, speech and hearing disorders, and a cleft lip, alveolus, and palate. They may also have extracranial anomalies, including heart and kidney defects and fused or missing vertebrae (which occur in $30 \%$ of cases). The resulting scoliosis causes the cranium to be obliquely positioned on the thoracic

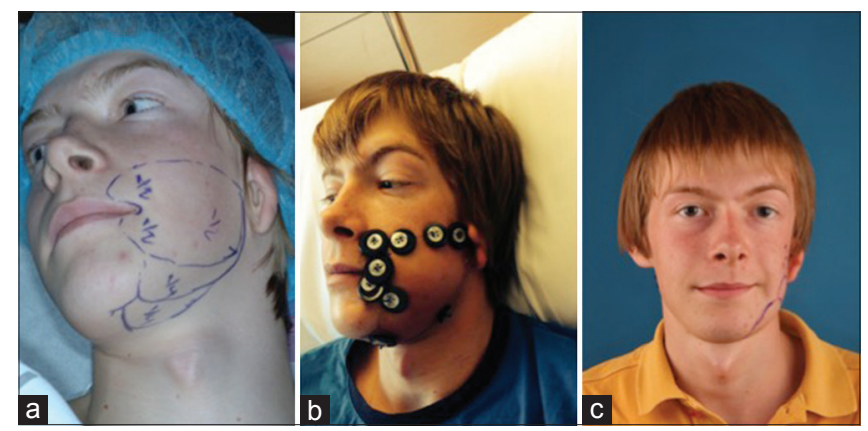

Figure 9: The same patient as in Figure 8 and one year after the facial rotation procedure. (a) Markings for free gluteal fat grafting, with access in front of the ear appendage; (b) immediate postoperative view, with buttons keeping the fat graft in position; (c) results one year after free fat grafting. Typically the fat graft has descended and requires tailoring via liposuction or lipofilling, which is marked on the skin

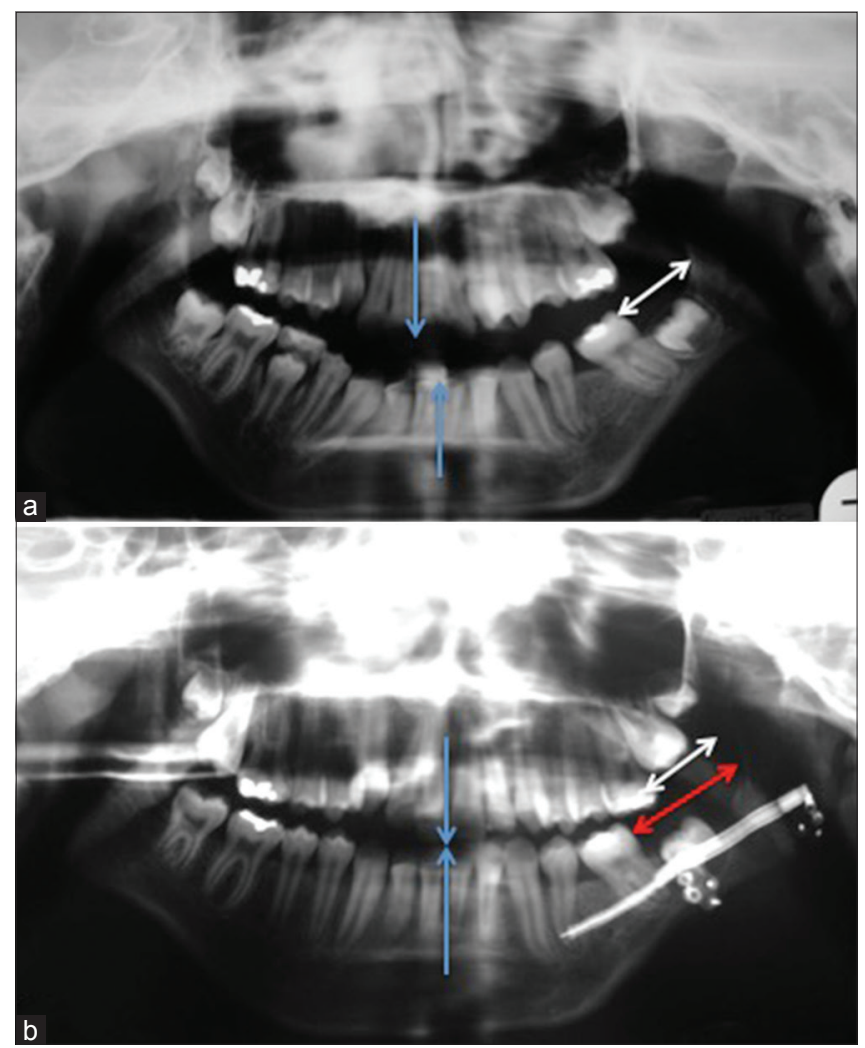

Figure 11: Pruzansky-Kaban Type Ila, illustrating the benefits of osteodistraction of the horizontal ramus. (a) Orthopantomogram prior to osteodistraction. Note the dental midlines are not aligned (blue arrows). The white arrow indicates the distance between the vertical ramus and the erupted last molar; (b) orthopantomogram immediately following osteodistraction. The dental midlines (blue arrows) are now aligned. The white arrow indicates the original and the red arrow represents the postdistraction distance (regenerate gain) between the ascending ramus and erupted last molar

spine [Figure 15]. In hemifacial microsomia, a missing, deformed, or dystopic orbit may already cause the normal reference frames (bipupillary plane, infraorbital plane, and brow plane) to be unreliable. When the patient is also scoliotic, the surgeon is challenged to find the best compromise for craniofacial symmetrization, as a completely symmetrical face may focus attention on an obliquely positioned head. In some instances, the orbital dystopia is striking and correctable with an orbital relocation osteotomy [Figure 16]. 


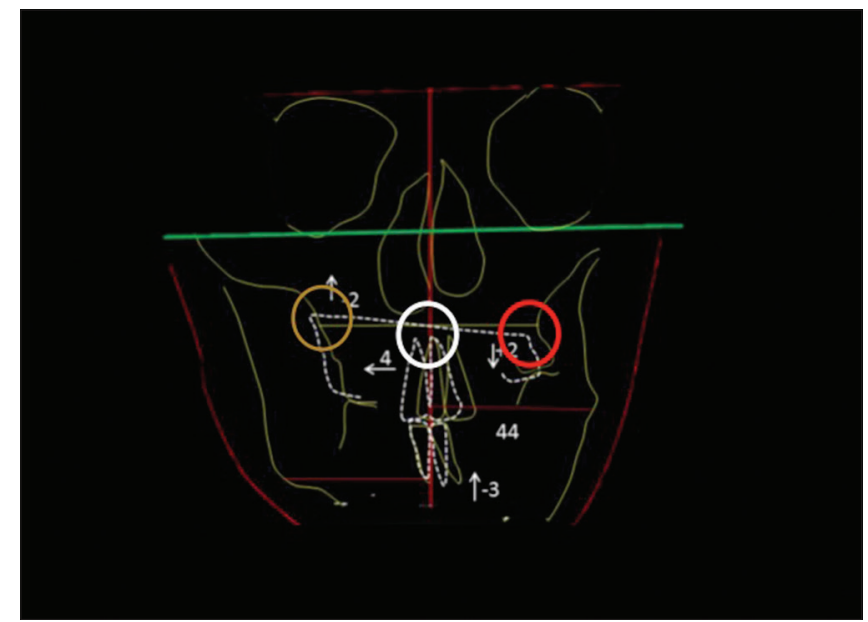

Figure 12: Choice of rotation pivot. The orange circle indicates impaction at the healthy side. The red circle indicates disimpaction at the affected side. The white circle indicates the pivot at the nasal spine
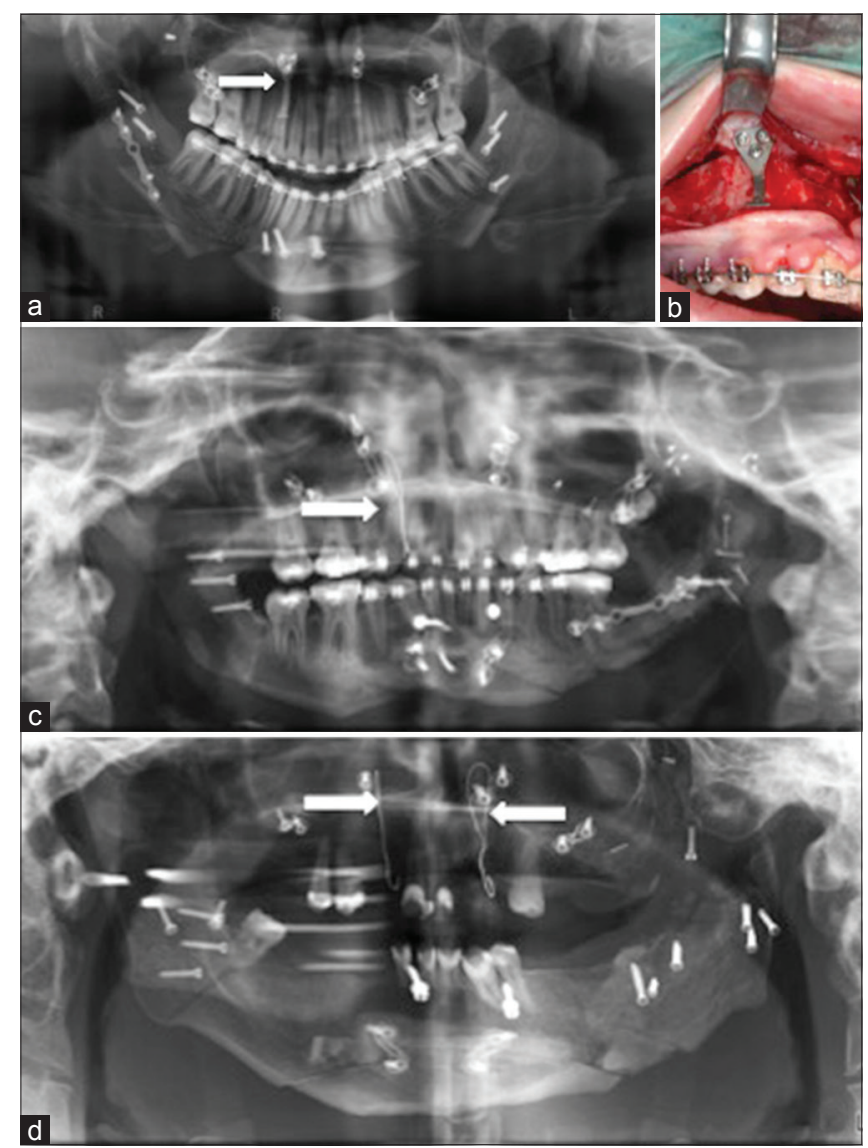

Figure 14: Skeletal suspension. (a) Orthodontic bone anchor (white arrow) placed at the affected side; (b) orthodontic bone anchor, which has to be removed via a mucoperiosteal flap dissection later on; (c) skeletal suspension by means of a piriform aperture skeletal wire $(0.5 \mathrm{~mm}$ diameter stainless steel wire, white arrow). The advantage of this technique is that the wire can be easily removed using local anesthesia, without flap preparation (http://www.scribd.com/doc/56442013/InterMaxillary-Fixation-Techniques-Manual); (d) bilateral piriform suspension (white arrows) in a case of massive mandibular advancement

\section{Point 6: Two costochondral grafts from ribs six} and seven

Two pieces of rib are required: one fully cartilaginous piece to reconstruct the fossa and one osseo-cartilaginous piece to reconstruct the missing condyle/ramus [Figure 17]. The zygomatic arch is reconstructed or reinforced by a cranial

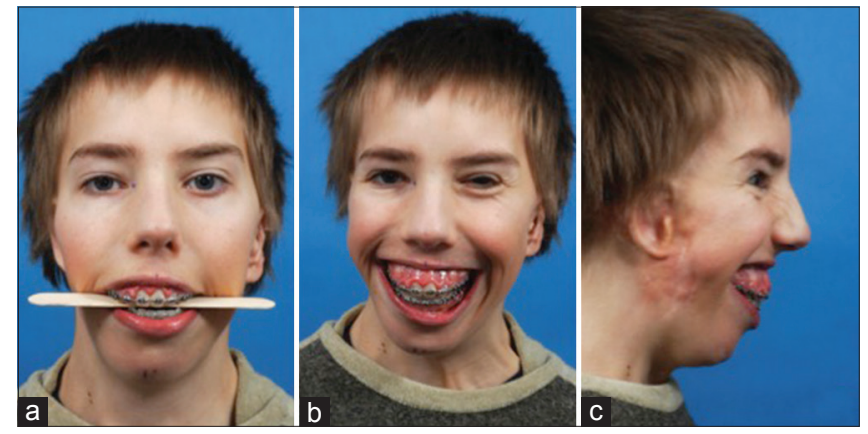

Figure 13: Pruzansky-Kaban Type IIb. This shows an ideal case with the horizontal occlusal plane in the maxilla and symmetrical gummy smile. A pivot was not chosen since overall impaction was required, with the translation of the dental midline to the left. (a) Frontal view, with the tongue spatula indicating a horizontal occlusal plane; (b) gummy smile in the frontal view; (c) gummy smile in the profile view

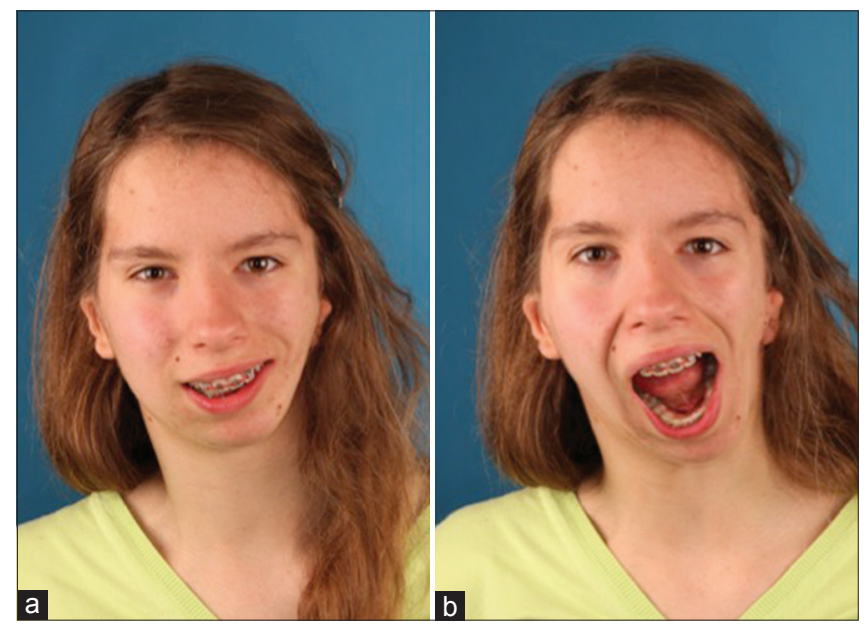

Figure 15: Pruzansky-Kaban Type IIb, after joint reconstruction with costochondral grafting. The patient has orbital facial nerve paresis, a small and displaced left orbit, cervical vertebral fusions and scoliosis, macrostomia and commissurala symmetry, microtia, and hearing and speech problems (Goldenhar syndrome). Because of the vertebral column problems, her neck is in an oblique position, and her head is off-center in relation to her body. Her head is positioned somewhat less obliquely than her neck. Her bipupillary plane is not horizontal. There is no drooping of the brow on the affected side, despite the facial nerve paresis. It is difficult to know which reference plane to choose for positioning the occlusal plane and maxillary dental midline. (a) Frontal view, natural head position; (b) frontal view, with normal mouth opening

bone graft. The cartilaginous piece is inserted behind the arch onto the skull base and is retained by resorbable sutures placed around the de novo zygomatic arch. The condylar replacement is fixed onto the ramus, using the temporal approach alone or in combination with an intraoral approach for Pruzansky-Kaban Type III. The cartilaginous part of the condylar replacement may be $1 \mathrm{~cm}$ high, as growth is allowed. ${ }^{[10]}$ Swinging the mandible to the healthy side is permitted during joint reconstruction, but it should not cause strain. The main objective is to create a functioning joint, normal range of mouth opening, and abutment allowing for a stable facial rotation procedure at a later age. When obtaining the rib grafts, it is important to remember that rib cartilage may be required for ear reconstruction as well.

Point 7: Pruzansky-Kaban Type Ilb reconstruction by a temporal approach

When only the upper part of the ascending ramus is absent, the craniofacial reconstruction (including calvarial 


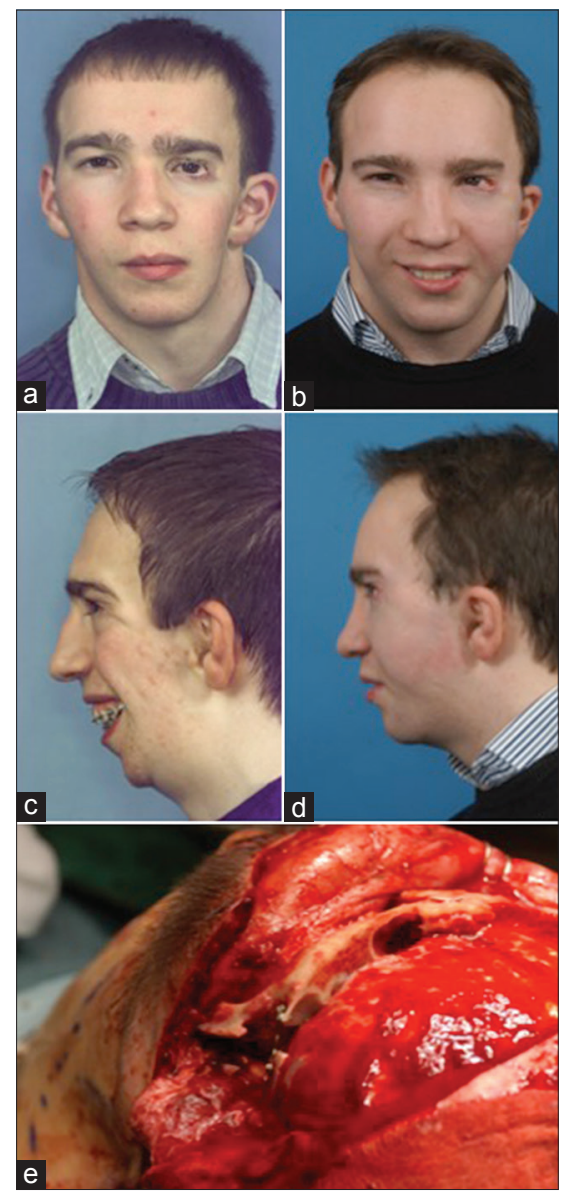

Figure 16: Goldenhar syndrome with scoliosis and orbital dystopia. The left orbit has been repositioned $1 \mathrm{~cm}$ higher. Free gluteal fat grafting, micro lipofilling, and a face-lift on the affected side were also performed after joint reconstruction and facial rotation. (a) Frontal view before the aforementioned procedures; (b) frontal view after the aforementioned procedures; (c) profile view before the aforementioned procedures; (d) profile view after the aforementioned procedures; (e) intraoperative view of the transcranial orbital repositioning (with the assistance of P. Staels, neurosurgeon)

bone harvesting, donor defect reconstruction, zygomatic arch and glenoid fossa reconstruction, and condylar reconstruction) can be performed via a single, wave line incision in the temporal region, extending to the lowest part of the auricular appendage [Figure 18]. Adding a retromandibular incision will jeopardize the facial nerve, as its location is abnormal. Adding an intraoral incision increases the risk of infection of the bone graft.

Point 8: Antero-medial reconstruction of the glenoid fossa versuspostero-lateral relocation of the joint

The issue in Pruzansky-Kaban Type IIb and III deformities is the location for the reconstruction of the joint. Creating an abutting joint in a location that has been determined by the anomalous development is easier; however, it is doubtful whether medial reconstruction will allow symmetrization of the midface and lateral mandible at a later stage [Figure 19]. Relocation of the joint to a mirrored position is more difficult in terms of the healing of the reconstructed condyle being transplanted obliquely to the mandibular stump. The composition is mechanically unstable when it assumes a $30^{\circ}$ angle in the frontal plane.
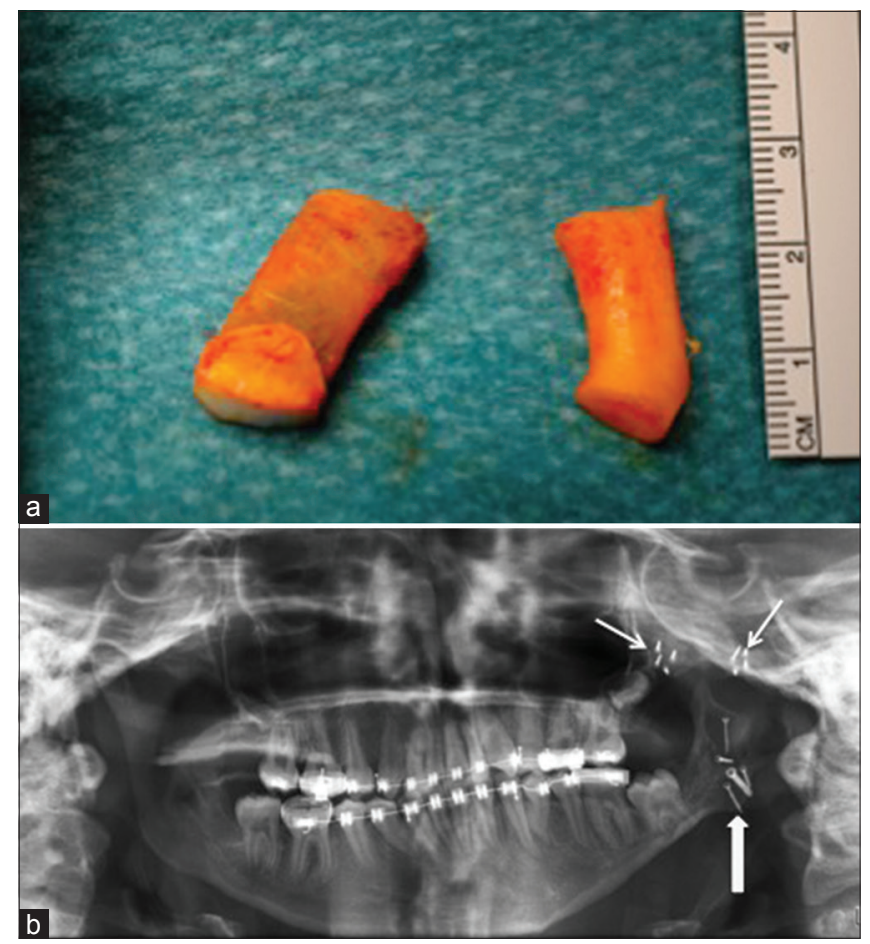

Figure 17: Pruzansky-Kaban Type IIb. Reconstruction of the temporomandibular joint. (a) Two harvested rib segments, one containing bony and cartilaginous components and the other containing only cartilage; (b) the zygomatic arch has been reconstructed using calvarial bone (two small arrows pointing at the micro osteosynthesis screws) and the fossa has been reconstructed using the cartilaginous segment (not visible). The condyle has been reconstructed using the costochondral segment, osteosynthetized via the temporal approach (big arrow pointing at oblique osteosynthesis screws)

Point 9: Respect the limits of lower facial advancement in favor of masticatory efficiency In extreme cases of sagittal deficiency such as that found in Pruzansky-Kaban Type III it is not necessarily desirable to advance the mandible into a position that will allow the soft tissue profile of the chin to be the ideal, as determined by Facewizz software (Orthoface $R$ and D, Sint-MartensLatem, Belgium) (www.facewizz.com). Such advancement will be opposed by the sphenomandibular ligament and the geniohyoid muscles, thereby jeopardizing the maintenance of occlusal stability. Maxillary, mandibular, and chin advancements should be tailored to the encountered strain. In these instances, chin augmentation with calcium phosphate paste (Hydroset, Stryker, Kalamazoo, MI, USA) can be helpful in increasing chin projection [Figure 20].

Point 10: Reconstruction of the lateral and posterior ramus with added manufacturing technology

Several options exist for augmentation of the lateral aspect of the ramus, but few exist for augmentation of the posterior aspect. For lateral augmentation, sliced lyophilized cartilage grafting can be an option if this is still available. Bone grafting may lead to resorption, and alloplastic implants may lead to extrusion after infection, hydroxyapatite granules mixed with fibrin glue provide a better option.

Both lateral and posterior augmentation are possible using 3D printed titanium, designed according to the postoperative computed tomography scans. For this purpose, the authors use ProPlan CMF and 3-matic 

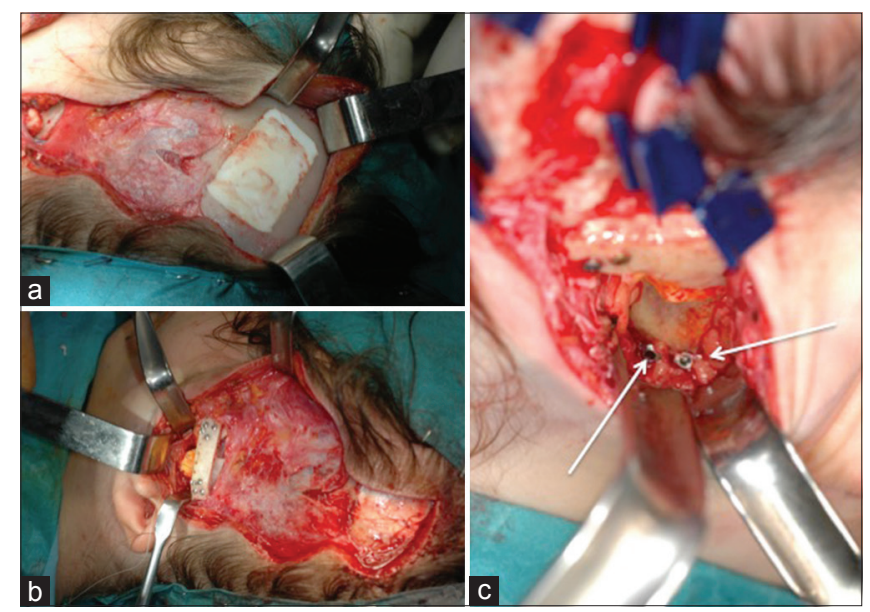

Figure 18: Pruzansky-Kaban Type IIb cases. A Temporal approach provides access to both the calvarial bone donor region and to the joint. Additional submandibular access is not required to osteosynthetize the costochondral graft to the ascending ramus. (a) The calvarial donor defect is reconstructed using calcium phosphate paste (Hydroset, Stryker); (b) the joint can be exposed and reconstructed in "open sky" mode; (c) two osteosynthesis screws have fixed the new condyle to the ascending ramus (arrows)
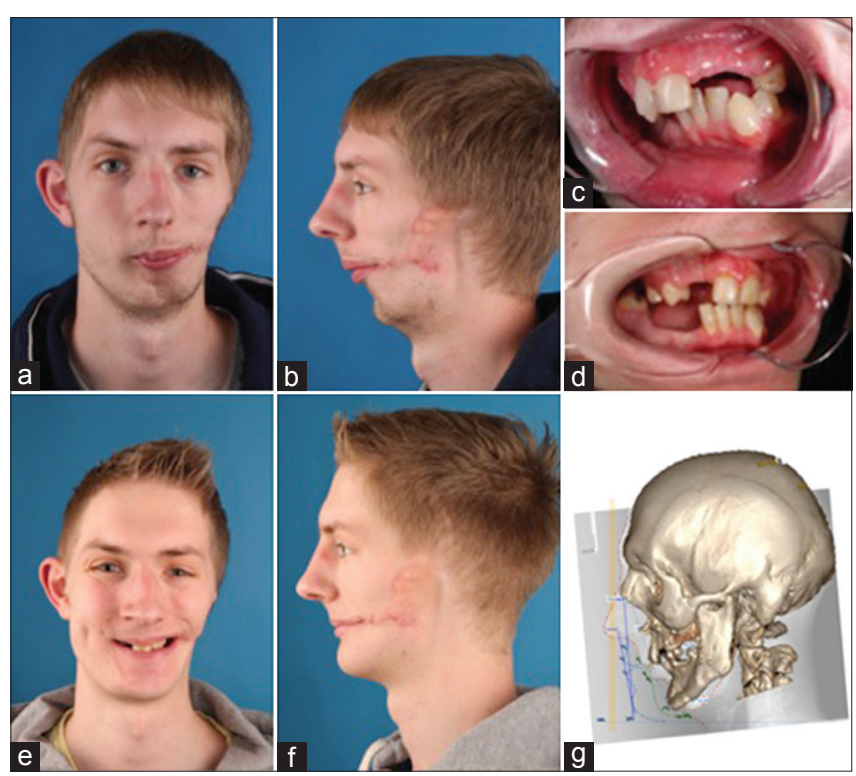

Figure 20: Pruzansky-Kaban Type III case undergoing a facial rotation procedure. The ideal profile line according to www.facewizz.com is coloured blue $(\mathrm{g})$. The targeted profile is colored green. (a) Frontal view, relaxed, before facial rotation; (b) left profile view, relaxed, before facial rotation; (c) three-fourths profile view of the dental occlusion, before facial rotation; (d) three-fourths profile view of the dental occlusion, after facial rotation. Proper prosthetic rehabilitation can be undertaken secondary to occlusal stability; (e) frontal view, smiling, after facial rotation; (f) profile view, relaxed, after facial rotation; (g) planning of the advancement. A three-dimensional (3D) computed tomography (CT) reconstruction is layered over the profile cephalogram, which was used to predict the ideal advancement (blue profile line) and the targeted advancement (green profile line), based on the risk of postoperative relapse and the consequences related to dental occlusion

software of Materialise (Heverlee, Belgium). Layerwise 3D-Systems (Heverlee, Belgium) prints the implants with porous bone interfaces and sandblasted soft tissue interfaces [Figures 21 and 22]. Two important questions during the design process are: (1) is the "normal" jaw angle excessively prominent and in need of reduction, and (2) will the soft tissue (e.g. masseter muscle) deficiency already be compensated for by adding hardware?
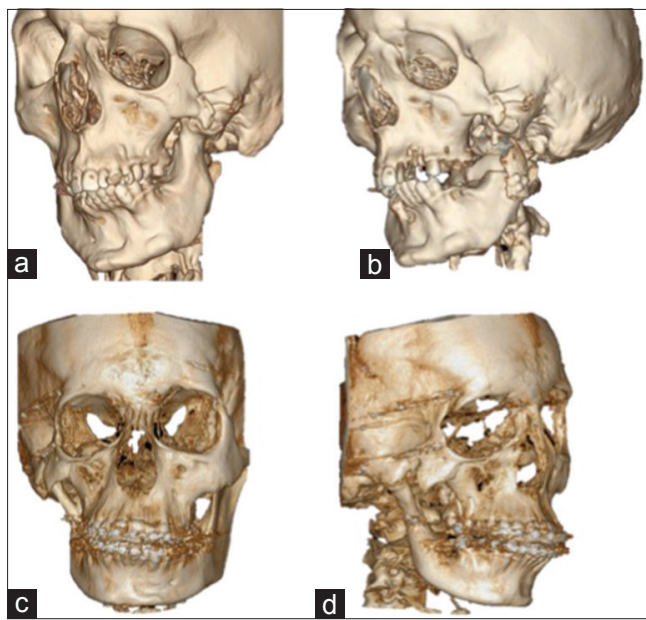

Figure 19: Pruzansky-Kaban Type III cases with joint reconstruction. (a) This patient underwent early joint reconstruction at the age of 4 but did not comply with physiotherapy and was lost to follow-up during the next 16 years. He returned with temporomandibular joint ankylosis and severe tooth decay; (b) the ankylosis was removed and a new costochondral graft was directed to the original fossa location; (c) frontal view immediately postoperatively of a patient who underwent late joint reconstruction. She had undergone surgery for plagiocephaly at a younger age. The joint was relocated more posteriorly and laterally. As a consequence, the rib graft was inclined at a $30^{\circ}$ angle to the ascending ramus. Healing and postoperative physiotherapy were uneventful. A mouth opening range of $37 \mathrm{~mm}$ was obtained with full graft union; (d) three-fourths right profile view of the case in (c)

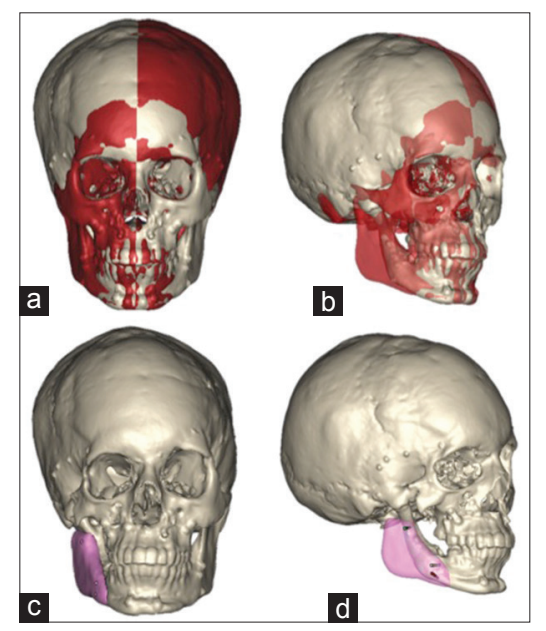

Figure 21: Pruzansky-Kaban Type III, following joint reconstruction and facial rotation. (a) Frontal view showing mirroring with ProPlan CMF. The red colored volumes are those with "normal" anatomy on the other side. Substantial vault asymmetry exists as this patient was also treated for plagiocephaly in the 1st year of live; (b) three-fourths right profile view. The transparency shows the underlying original. Nonetheless, it is hoped that the comprehensive treatment planning described in this report may be used to promote optimal patient care ascending ramus; (c) frontal view. Implant design in pink; (d) three-fourths profile view. Transparent implant design indicates the fixation screws

\section{DISCUSSION}

The vertical ramus compartment in hemifacial microsomia can exhibit variable degrees of hard or soft tissue deficiencies. Growth and development result in distorted proportions in both the transverse and sagittal dimensions. Surgical correction is challenging with respect to decision-making and execution, but is nonetheless highly rewarding. Older strategies have been tackled by newer technologies. The author has witnessed the rise in 


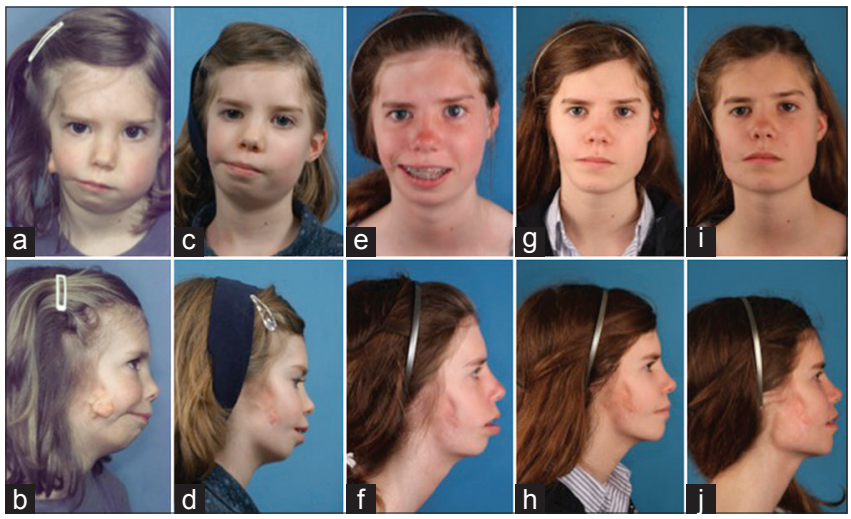

Figure 22: The same patient as in Figure 20. (a and b) Before joint reconstruction; (c and d) after joint reconstruction; (e and f) before facial rotation, after orthodontic preparation; ( $\mathrm{g}$ and $\mathrm{h}$ ) after facial rotation; (i and $\mathrm{j}$ ) after three-dimensional (3D) titanium print implantation of the right mandible
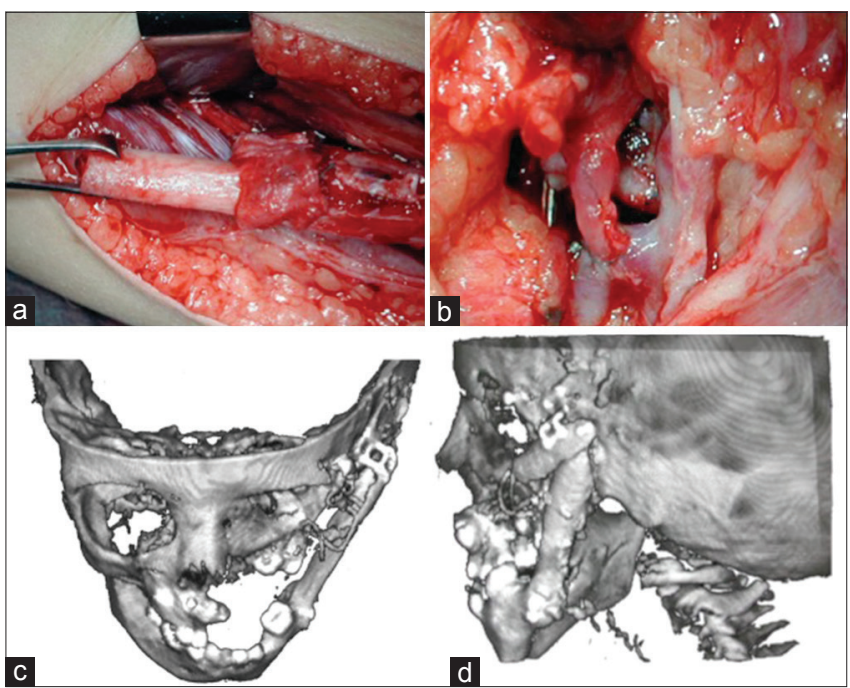

Figure 23: Pruzansky-Kaban Type III. Microvascular fibula transfer in a 10-year-old patient. (a) Harvesting of the fibula; (b) microvascular anastomosis; (c) three-dimensional (3D) computed tomography (CT) reconstruction after fibula transplantation to the left mandible; (d) 3D $\mathrm{CT}$ reconstruction, profile view

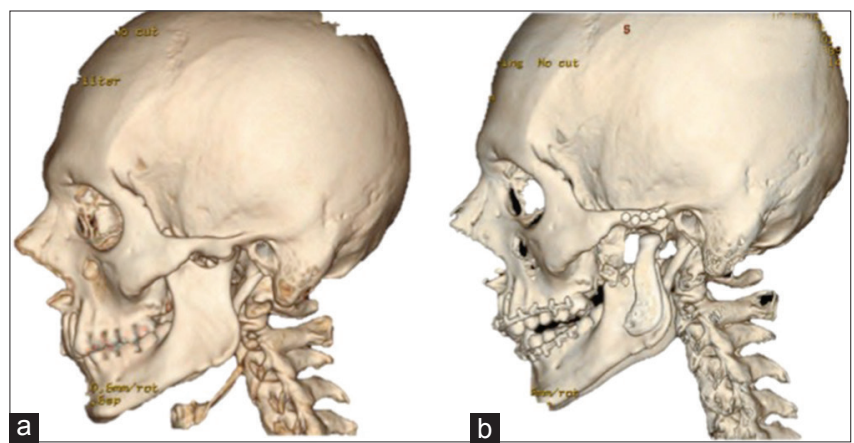

Figure 24: Bilateral three-dimensional printed temporomandibular joint (TMJ) prostheses. (a) TMJ ankylosis present; (b) fossa/condyle prosthesis in place

popularity of 3D early osteodistraction, ${ }^{[11,12]}$ and then its subsequent fall in use. ${ }^{[13]}$ Microvascular fibula transfer in a young patient ${ }^{[14]}$ [Figure 23] has been used, but the author has returned to nonvascularized rib transplantation. ${ }^{[15]}$ Microvascular parascapular dermofat transfer ${ }^{[16]}$ has been abandoned by the author in favor of nonvascularized gluteal fat transfer. ${ }^{[17]}$ The author has also used 3D printed condyle/fossa reconstruction in adults with ankylosis ${ }^{[18]}$ [Figure 24] with consideration for integration in the facial rotation procedure for hemifacial microsomia following rib graft failure.

This article represents level 5 evidence, and therefore simply provides an expert opinion. The variability of pathology, lack of a gold standard, different surgical experiences, duration of the phased treatment, desiderata, compliance and economic situation of the patients, and use of new technologies prohibit valid sampling and prospective analyses. Nonetheless, it is hoped that the comprehensive treatment planning described in this report may be used to promote optimal patient care.

\section{REFERENCES}

I. Grabb WC. The first and second branchial arch syndrome. Plast Reconstr Surg 1965;36:485-508

2. Vento AR, LaBrie RA, Mulliken JB. The O.M.E.N.S. classification of hemifacial microsomia. Cleft Palate Craniofac J I991;28:68-76.

3. Pruzansky S. Not all dwarfed mandibles are alike. Birth Defects 1969;5: 120-9.

4. Kaban LB, Moses MH, Mulliken JB. Surgical correction of hemifacial microsomia in the growing child. Plast Reconstr Surg 1988;82:9-19.

5. Harvold EP, Vargervik K, Chirici G. Treatment of Hemifacial Microsomia. New York: Alan R. Liss; 1983.

6. Kaban LB, Moses MH, Mulliken JB. Correction of hemifacial microsomia in the growing child: a follow-up study. Cleft Palate J 1986;23 Suppl 1:50-2.

7. Mommaerts MY, Nagy K. Is early osteodistraction a solution for the ascending ramus compartment in hemifacial microsomia? A literature study. J Craniomaxillofac Surg 2002;30:20I-7.

8. Nagy K, Kuijpers-Jagtman AM, Mommaerts MY. No evidence for long-term effectiveness of early osteodistraction in hemifacial microsomia. Plast Reconstr Surg 2009; |24:206|-7|.

9. Obwegeser HL. Correction of the skeletal anomalies of oto-mandibular dysostosis. J Maxillofac Surg 1974;2:73-92.

10. Peltomäki T. Growth of the costochondral junction and its potential applicability for the reconstruction of the mandibular condyle. Turku: Turunyliopisto; 1993.

II. Molina F, Ortiz Monasterio F. Mandibular elongation and remodeling by distraction: a farewell to major osteotomies. Plast Reconstr Surg 1995;96:825-40.

12. Ortiz Monasterio F, Molina F, Andrade L, Rodriguez C, Sainz Arregui J. Simultaneous mandibular and maxillary distraction in hemifacial microsomia in adults: avoiding occlusal disasters. Plast Reconstr Surg 1997; I00:852-6I.

13. Kunz C, Brauchli L, Moehle T, Rahn B, Hammer B. Theoretical considerations for the surgical correction of mandibular deformity in hemifacial microsomia patients using multifocal distraction osteogenesis. J Oral Maxillofac Surg 2003;61:364-8

14. Stelnicki EJ, Boyd JB, Nott RL, Barnavon Y, Uecker C, Henson T. Early treatment of severe mandibular hypoplasia with distraction mesenchymogenesis and bilateral free fibula flaps. J Craniofac Surg 200I;12:337-48.

15. Lee SJ, Lee HP, Tse KM, Cheong EC, Lim SP. Computer-aided design and rapid prototyping-assisted contouring of costal cartilage graft for facial reconstructive surgery. Craniomaxillofac Trauma Reconstr 2012;5:75-82.

16. Tanna N, Broer PN, Roostaeian J, Bradley JP, Levine JP, Saadeh PB. Soft tissue correction of craniofacial microsomia and progressive hemifacial atrophy. J Craniofac Surg 2012;23:2024-7.

17. Lim AA, Fan K, Allam KA, Wan D, Tabit C, Liao E, Kawamoto HK, Bradley JP. Autologous fat transplantation in the craniofacial patient: the UCLA experience. J Craniofac Surg 2012;23:1061-6.

18. Haq J, Patel N, Weimer K, Matthews NS. Single stage treatment of ankylosis of the temporomandibular joint using patient-specific total joint replacement and virtual surgical planning. Br J Oral Maxillofac Surg 20।4;52:350-5.

How to cite this article: Mommaerts MY. Hemifacial microsomia: management of the vertical ramus compartment. Plast Aesthet Res 2015;2:99-106.

Source of Support: Nil, Conflict of Interest: None declared.

Received: 01-02-2015; Accepted: 07-04-2015 